\title{
Diabetes technology
}

\author{
THOMAS SJ CRABTREE ${ }^{1,2,3}$, TIM STREET ${ }^{4}$, EMMA G WILMOT ${ }^{1,2}$
}

Figure 1. Timeline showing development of diabetes technologies with some commonplace gaming and mobile telephone technologies for comparison. ${ }^{10,11}$

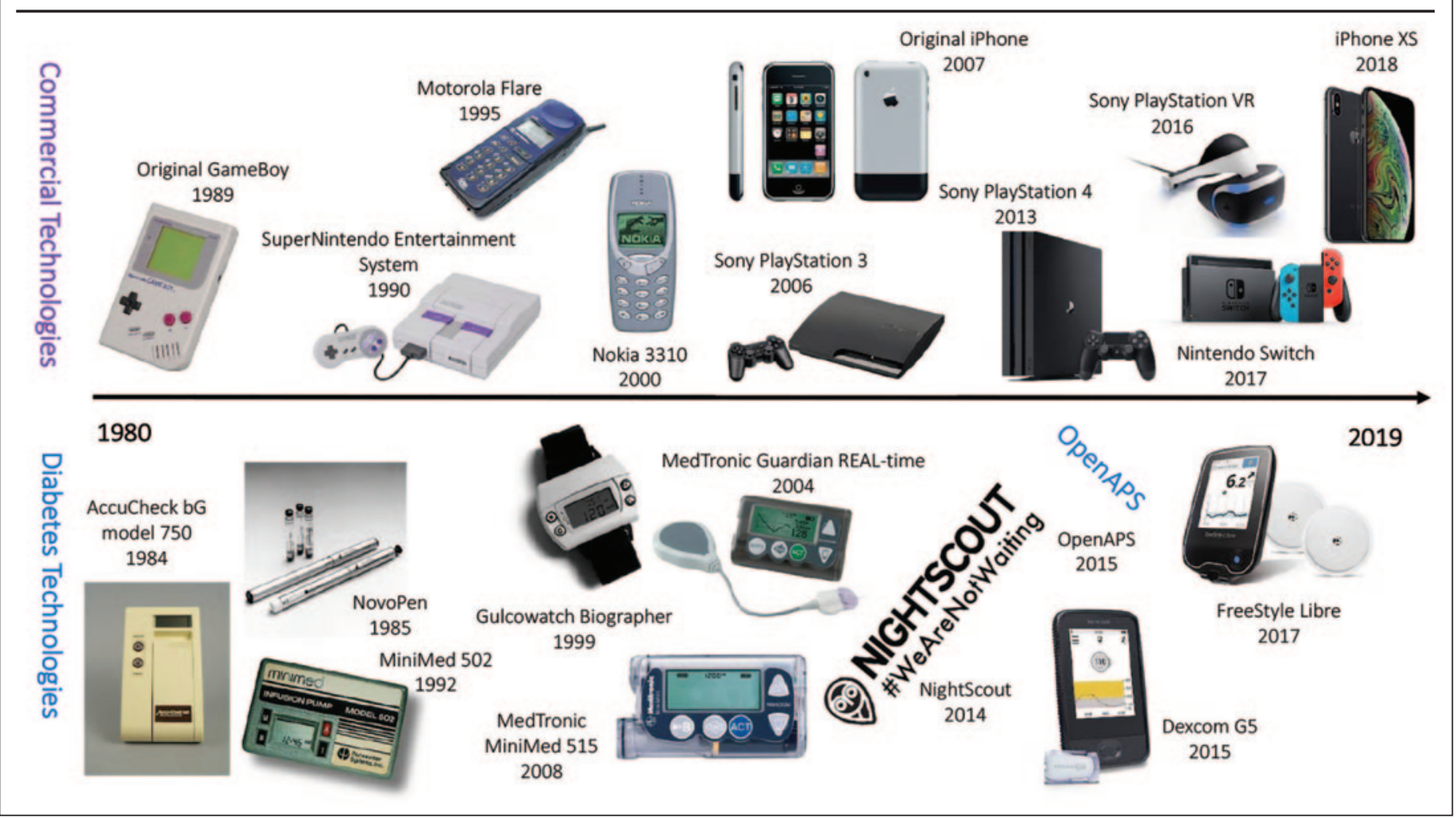

Key words: diabetes, technology, do-it-yourself artificial pancreas, patient experience

\section{Introduction}

Type 1 diabetes can be a challenging condition to manage. Recent developments in diabetes technologies and their increasing availability have had a positive impact on the lives of people with dia-

University Hospitals of Derby and Burton NHS Trust, Derby, UK

2 University of Nottingham, UK

Sandwell and West Birmingham Hospitals NHS Trust, Birmingham, UK

DIY APS User

Address for correspondence: Dr Thomas SJ Crabtree Clinical Research Fellow, Derby Teaching Hospitals NHS Foundation Trust, Department of Diabetes \& Endocrinology, Royal Derby Hospital, Uttoxeter Road, Derby, Derbyshire, DE22 3NE

E-mail: T.Crabtree@nhs.net

Br J Diabetes 2019;19:136-140

https://doi.org/10.15277/bjd.2019.231 betes. There are data which suggest that glucose monitoring technologies such as continuous glucose monitoring and flash glucose monitoring are associated with improvements in glucose control and treatment satisfaction. ${ }^{1,2}$ Recently, the first commercially available hybrid closed loop system (consisting of an insulin pump, continuous glucose monitoring and an algorithm to automate insulin delivery) entered the market. However, such technologies require stringent regulatory approvals and, as such, can be slow to develop and, as the person below reflects, this is often much slower than commercial technologies and gaming systems (see timeline in Figure 1). A tech-savvy generation of people with diabetes has been driving forward the development of 'artificial pancreas systems' (APS) (\#WeAreNotWaiting) without input from traditional technology companies or regulatory approval. From a healthcare professional perspective, that can produce problems.

Below is an adaptation of a piece written by someone living with diabetes, his reflection on current diabetes technologies, what the future may hold (including APS) (full unedited version 
available on request) and discussions from diabetes professionals with an interest in technology on where the evidence lies, what the ethical and medico-legal considerations are, and how we can support people with diabetes who are opting to use unregulated and unapproved devices, should we wish to do so.

\section{Diabetes technology: a personal perspective} Tim Street

Email: tim@diabettech.com

Looking at diabetes technology, some would argue that, as a person with type 1 diabetes, I've never had it so good: the ability to monitor glucose levels in real time, some of the fastest acting insulins on the market and automated insulin delivery systems from vendors that can provide much better management of diabetes. Yet I would argue that they are missing the point, and that in reality the state of diabetes technology right now is 'so-so', and while progress is being made, there is plenty still to do.

I was diagnosed with type 1 diabetes in 1988 at a time when consumer technology was just getting exciting. At the time, diabetes technology for me was Human Protophane insulin (or NPH as it's probably better known) twice a day and BM 1-44 test strips which, when you went into hospital, were put into an optical meter as we did not have one at home because they were hundreds of pounds each. With my parents being a teacher and a vicar, we didn't really have that kind of cash. The coolest diabetes gadget I had set eyes on as a 13-year-old was the Novopen, owned by the registrar who was using it for multiple daily injections of Actrapid (Novo Nordisk).

The next 10 years could have been considered as the forgotten years of diabetes technology, with very little change obvious in the technologies with which diabetes was managed and treated, but noticeable (perhaps retrograde) steps in management techniques.

After 20 years had passed, pumps had become smaller and more easily available, and there was now continuous glucose monitoring (CGM) with sensors that lasted for 3 days and were notoriously inaccurate. Faster acting rapid insulins were in the mainstream, as were better longer acting insulins that were less likely to cause low glucose levels due to a flatter absorption profile. Blood glucose meters had improved, taking only 10 seconds to provide a result instead of the 120 seconds when I was first. diagnosed. But Diabetes Tech was struggling to keep up with Consumer Tech.

Over the next 10 years things changed considerably. By 2018, when I had been diagnosed for 30 years, CGM had become more widely available in some parts of the world and was considerably more accurate and reliable, even while remaining too expensive for most people, while pumps got more ubiquitous in certain territories. Flash monitoring had arrived, and various start-ups pledged their desire to develop and sell 'artificial pancreas systems' (APS).

Until around 2015 one might argue that commercial companies developing tools for use with diabetes were not really inter- ested in the most important factor - the person at the centre of the system desperate for technology support which was not developing quickly enough. This resulted in a group of incredibly talented engineers, scientists and software developers individually working out how to extract data from devices and use it in far more effective ways, which culminated in what we now know as NightScout.

\section{User-driven innovation and DIY diabetes technology} NightScout is a glucose cloud storage system which was developed for Dexcom. CGM allowed parents to remotely keep track of their children's glucose levels, a revelation in terms of diabetes care, but something that did not appear to be appreciated in the Med Tech world.

This became the start, as tools were created that allowed the CGM systems to talk to telephones and upload to be handled wirelessly and conveniently, leading to DIY CGM software that captured the data and offered far greater flexibility than the tools that vendors were providing. The lack of interoperability provided by commercial tools had not gone un-noticed and was being addressed by those who had the skills and desire to do so.

At around the same time, Ben West had spent 6 years decoding the communication between older Medtronic pumps, while Dana Lewis and Scott Leibrand had been working on how to make her CGM alarms louder and predict future glucose levels. In 2015, after the culmination of their work, OpenAPS was released to the world as a toolkit to build an APS, complete with its core algorithm Oref0 (which was succeeded by Oref1 in 2018).

It was quickly followed by Loop in 2016, a similar system with a different algorithm that ran on an iPhone, using the RileyLink, a radio communications device that converted Bluetooth instructions into the $916 \mathrm{mHz}$ radio signals the pump understood, designed by Pete Schwamb, and that listened in to the Dexcom G5 communications with the iPhone Dexcom app and provided them to Loop to use. Coming, as it did, from Nate Racklyeft, an Apple developer, it also has a beautiful user interface. (In early 2019, connectivity was established with the Omnipod Eros series, enabling more people to use the system with in-warranty pumps).

Finally, in 2017, Milos Kozak, along with a number of other developers (including Adrian Tappe) encapsulated the OpenAPS algorithm into an Android application known as AndroidAPS, a modular system that used plug-ins to allow multiple different pump drivers to be created and that could consume CGM data from a number of different sources. This has proved to be extremely popular throughout Europe as it provided compatibility with, initially, the DanaR pump from Sooil, then saw development of drivers for the Sooil DanaRS, Roche Spirit Combo and Insight pumps, which had wide use and, latterly, the same Medtronic pumps that the other two systems have access to, plus the Omnipod.

These systems are now used by thousands of people worldwide. The CGM in the Cloud Facebook group, which is the home of NightScout support, has over 30,000 members. Amongst those who have reported that they use the DIY APS, it is estimated that 
there are over 12 million looping hours (a number that is updated regularly at https://www.openaps.org/outcomes), and even this is believed to be a gross underestimation. ${ }^{3}$

When taken in context, this is significantly larger than the number of loop hours for pivotal trials that are presented to the Food and Drugs Administration (FDA).

\section{What does the future hold?}

In early 2018, if you looked carefully at what was going on you would have expected multiple single vendor systems where you were locked into an algorithm/pump/sensor combination with no way of knowing how biocompatible you were with each one and no way of picking the best components for you. However, in an effort to speed up certification of medical devices in the USA, the FDA created a componentised certification model, where each of the individual items had to have some additional 'interoperability' functions that would allow each item in the chain to understand the status of what it was communicating with.

This generated the following designations:

- iCGM

- ACE pump (Alternate Controller Enabled; formerly iPump)

- iController

This opened up the ecosystem and allowed an individual company to focus on what it was good at. As a result, during 2018, Tidepool announced that they would be producing a version of Loop, to be known as Tidepool Loop, that would work with the Omnipod Dash system (which has received an ACE designation) and be approved by the FDA as an iController. In June 2019 they took this a stage further and announced that they would also partner with Dexcom and Medtronic with a new Bluetooth-enabled pump and sensor, which would have ACE and iCGM designation, respectively. From an end-user perspective, this opens up the game. Now there will be options to select something that is not locked into a single vendor and, as an end-user, I hope there will be more iControllers that will offer end-users the opportunity to pick what works best for them, rather than being told by manufacturers or those who are holding the purse strings.

\section{Technology isn't the entire answer}

Prior to using a DIY APS I had 'sugar surfed', which entailed checking CGM readings every 20 minutes or so and taking an action, but it also required regular review and care overnight when glucose levels could drop after exercise. Using these technologies saves me a huge amount of time and effort as I no longer have this overhead of checking and reacting. Sure, I sometimes watch it do its thing out of fascination; however, it means that a high time in range and low $\mathrm{HbA}_{1 c}$ require next to no effort on my part. The other huge benefit is knowing that it doesn't really matter what time I eat at night or whether I have exercised; I know that I will wake up in range the following morning without really having to do anything to get there.

However, as much as I - as a user of a DIY closed loop system - might enjoy what it offers me and think it makes a massive dif- ference to my ability to manage diabetes, it is not the only requirement for people living with diabetes. There are two other areas that need consideration if we are to look at technology being useful. The first of these is education.

While technology is amazing and can be life changing, without a basic education of how to live with diabetes there is a need to comprehend the issues that technology can cause when it goes wrong. Equally, without appropriate education it is almost impossible to make an informed decision as to what you should select to make living with diabetes easier.

But education is also required for those in healthcare who are tasked with assisting people with diabetes to make these decisions. They need to understand not only the technologies and what they offer, but also the human beings at the core of the decisions and what would allow them to live their lives to the fullest.

The second is access. As much as we can talk about technology and the effects it can have, if people cannot get hold of it and use it, it is worthless. Whether it is DIY or a commercial system, without access you have, essentially, nothing.

In DIY terms, that means access to CGM and pump systems, as the controller is free to access. However, within the UK model, CGM is extremely difficult to obtain through the NHS, at least if you're an adult. If you look at the commercial systems the costs go up again, and now (without Tidepool Loop) the entire system is priced at a level such that only those who are in greatest need get a look in.

Both these examples highlight the difficulties faced by healthcare systems in relation to APS. Unless a mechanism can be found to bring down the fundamental cost, then healthcare systems will struggle to find ways to pay for what is on offer.

With reference to the title of this article - that this is a personal perspective of diabetes technology - there are three points that summarise my views:

- Using a DIY APS system has made living with type 1 diabetes a completely different experience; no longer is it necessary for me to keep part of my brain focused on it. Instead, my technological friend watches and manages what's going on, leaving me to get on with life and allowing me to spend more time on other things and less on type 1 diabetes. Everyone with type 1 diabetes should have access to this type of assistance.

- Diabetes technology has been very slow in keeping up with the expectations of consumers, and often missed the point that those consumers should be at the heart of both the product and the process around that product; user-driven innovation has placed the user squarely in the fore and forced commercial providers and regulators to re-assess what they offer and how they offer it, encouraging innovation and progress.

- The future of diabetes technology looks significantly better than its past. Recognition of the importance of the consumer in the process and the acceptance of those responsible for user-driven innovation into both commercial and academic diabetes technology circles is driving significant change. 


\section{Diabetes technology: the professional perspective \\ Thomas Crabtree \\ T.Crabtree@nhs.net}

This story is a reminder of why technology is so important to the diabetes community. Things have definitely come so far and what many have aspired to - a fully automated closed-loop system - seems entirely tangible with the advent of do-it-yourself artificial pancreas systems (DIY APS). But they are right - there is much further to go and technology in isolation is not by any means the answer.

These systems all use approved and regulated devices such as FreeStyle Libre, Dexcom G6 and numerous different insulin pumps in a combination that is thus far unregulated and unapproved. People with diabetes who use these systems claim a high level of safety and consider that the constituent parts are all doing what they were designed to do.

As mentioned above, there are millions of hours of looping data available with little evidence of adverse outcomes. ${ }^{3}$ The FDA issued an alert about an adverse event outside the UK. However, we need to bear in mind that type 1 diabetes management is inherently risky with $8 \%$ of people reportedly having an episode of severe hypoglycaemia on an annual basis. ${ }^{4}$ Even regulated hybrid closed-loop systems carry warnings about the potential side effect of severe hypoglycaemia. The risks of DIY systems in comparison to commercially available systems are unknown and further data are required.

Outcomes for patients using DIY APS, although self-reported, are good with improved time-in-range and reductions in $\mathrm{HbA}_{1 c}, 3,5,6$ Users also report improvements in quality of life and satisfaction with the systems - in fact, this is probably the main reason many opt to use these systems. ${ }^{5}$ For anyone who has encountered a user in clinic, they will recognise the transformation that happens once the person is established on DIY APS and the reports of "I don't have to think about my diabetes all the time anymore", which can be life changing.

The author of this piece is also clearly aware of the need for education - DIY APS cannot be given to anyone to use and be expected to be safe and effective. Without knowing the underlying principles of diabetes management, carbohydrate counting and even the basics of pump safety, a person with diabetes would likely be at increased risk of an adverse outcome. However, would this be a greater risk than using pump therapy (in a non-DIY APS system) without appropriate education? The answer is probably not, but we have no evidence directly comparing the two, even when education is appropriately given.

DIY APS is also an ethical and medico-legal quagmire. As the systems are unapproved and unregulated, there are concerns that they may affect professionals who continue to provide pumps and consumables for their patients knowing they will be used for DIY APS. If the user comes to harm, where does the liability lie? If there is a problem with the pump, will it void the warranty? If a medical professional feels unable to provide consumables due to the concerns above, then, while arguably justified, it risks a deterioration in the patient-provider relationship with potentially even greater consequences. The balance is difficult to strike.

Position statements from the Juvenile Diabetes Research Fund ${ }^{7}$ and Diabetes $U K^{8}$ encourage choice for the person living with diabetes but advise caution in promoting the use of these systems. Both advise counselling users of the potential risks associated with these systems, but neither actively suggest discontinuing consumables or refusing pump renewals for people who would otherwise have been granted them.

The Diabetes Technology Network UK recently carried out a survey of healthcare professionals, the results of which were delivered in brief and are hoped to be published in full shortly. This demonstrated that many healthcare professionals recognise these devices can be "risky in the wrong hands" but then, ultimately, they are keen to support users who opt to use these systems. They also recognise, as does the author of this piece, that healthcare professionals also need education in this often complex technological system. Many feel ill-equipped and anxious dealing with something that they feel they know so little about.

Diabetes healthcare professionals can support the people with diabetes under their care. Diabetes UK has endorsed in its statement that all teams caring for known loop users register and take part in the ABCD nationwide DIY APS audit which will hopefully be launching in 2019/20. It is hoped this will provide objective observational data on the safety and effectiveness of DIY APS, providing the basis for future recommendations.

It could be argued that DIY APS will fade now that there are commercially available approved systems such as the Medtronic 670G. However, cost means that availability is limited and ultimately functionality is still lacking compared with some of the more advanced parts of the closed-loop algorithm such as unannounced meals and super-micro boluses. DIY APS has far from seen its day and, with a recent increase in compatible pump availability, sales of RileyLink devices (a component part of certain APS systems) have skyrocketed. ${ }^{9}$ Future DIY APS use is predicted to be much greater than it is now.

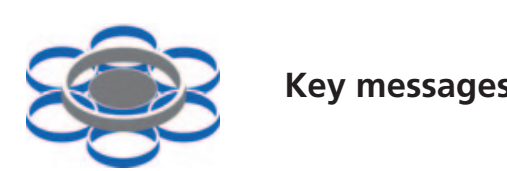

- Technology play a fundamental role in most of our day-to-day lives, but plays an even greater role in 2019 diabetes care

- Technology in isolation is not the key however education is!

- Do-it-yourself artificial pancreas systems and other advanced diabetes technologies are being taken up at a rapid rate by the diabetes community and we must be prepared to manage the use of these devices in our clinics in the decades ahead 
While healthcare professionals cannot recommend unregulated and unapproved systems, we can encourage honest conversations, counselling about the potential risks while continuing to support user choice if an informed decision is made for the individual to continue DIY APS at their own risk. There is still far to go. None of these are a cure, and it is our responsibility to educate and prepare ourselves for the wave of technology that is about to break upon our services.

This is an accompanying article of a talk given at the Royal Society of Medicine in London on 13 June 2019 on "New technologies in diabetes".

\section{Conflict of interest None Funding None}

\section{References}

1. Olafsdottir AF, Attvall S, Sandgren U, et al. A clinical trial of the accuracy and treatment experience of the flash glucose monitor FreeStyle Libre in adults with type 1 diabetes. Diabetes Technol Ther 2017;19(3):16472. https://doi.org/10.1089/dia.2016.0392

2. Edge J, Acerini C, Campbell F, et al. An alternative sensor-based method for glucose monitoring in children and young people with diabetes. Arch Dis Child 2017;102(6):543-9. https://doi.org/10.1136/archdischild2016-311530

3. OpenAPS Community. OpenAPS Ouctomes. Available at: https://ope- naps.org/outcomes/ (accessed 2 October 2019).

4. Pettus JH, Zhou FL, Shepherd L, et al. Incidences of severe hypoglycemia and diabetic ketoacidosis and prevalence of microvascular complications stratified by age and glycemic control in U.S. adult patients with type 1 diabetes: a real-world study. Diabetes Care 2019 Sep 23 [Epub ahead of print]. https://doi.org/10.2337/dc19-0830

5. Litchman ML, Lewis D, Kelly LA, Gee PM. Twitter analysis of \#OpenAPS DIY artificial pancreas technology use suggests improved A1C and quality of life. J Diabetes Sci Techno/ 2019;13(2):164-70. https://doi.org/ 10.1177/1932296818795705

6. Lewis D, Leibrand S, \#OpenAPS Community. Real-world use of open source artificial pancreas systems. J Diabetes Sci Technol 2016;10(6): 1411. https://doi.org/10.1177/1932296816665635

7. Juvenile Diabetes Research Fund. JDRF's UK position statement on type 1 diabetes 'DIY' technologies. 2019. Available at: https://jdrf.org.uk/ about-us/position-statements-reports/position-statements/jdrfs-uk-position-statement-on-type-1-diabetes-diy-technologies/ (accessed 2 October 2019)

8. Diabetes UK. Do it yourself (DIY) closed loop for people living with type 1 diabetes. 2019. Available at: https://www.diabetes.org.uk/resourcess3/2019-08/DIY\%20closed\%20looping\%20for\%20Type\%201\%20diabetes\%20position\%20statement.pdf.

9. Lucas J. Post re: RileyLink MedTronic/Ominipod sales data; Looped Facebook Page. Facebook, 2019

10. NovoNordisk. NovoNordisk - our history. Available at: https://www novonordisk.com/about-novo-nordisk/novo-nordisk-history.html (accessed 2 October 2019).

11. Hirsch IB. History of glucose monitoring. 2018. Available at: https://professional.diabetes.org/sites/professional.diabetes.org/files/media/db2018 11.pdf.

\section{ADCD \\ Association of tritish Clinical Diabotologists \\ FreeStyle Libre Nationwide Audit in progress \\ $A B C D$ is running a Nationwide Audit of FreeStyle Libre in real clinical use in the UK Does your centre use FreeStyle Libre?

\author{
If yes, REGISTER YOUR CENTRE! \\ http://www.diabetologists-abcd.org.uk/n3/FreeStyle_Libre_Audit.htm
} \\ - you are invited to enter your patients' data into the bespoke on-line tool \\ - you can collect data on the easy-to-complete paper proformas which you can acquire through the above web address \\ - you are able to analyse your local data easily \\ - the data will be automatically added to the national data in anonymised form \\ Please remember: \\ - the more data, the more complete our understanding of FreeStyle Libre in real clinical practice - all contributors will be listed in publications arising from data submission}

